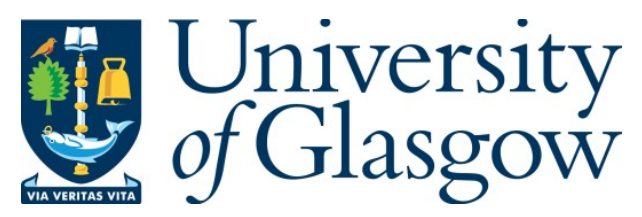

Zhang, J., Evans, B., Imran, M. A., Zhang, X., and Wang, W. (2016) Green Hybrid Satellite Terrestrial Networks: Fundamental Trade-Off Analysis. In: 2016 IEEE 83rd Vehicular Technology Conference (VTC Spring), Nanjing, China, 15-18 May 2016, ISBN 9781509016983.

There may be differences between this version and the published version. You are advised to consult the publisher's version if you wish to cite from it.

http://eprints.gla.ac.uk/133063/

Deposited on: 20 December 2016

Enlighten - Research publications by members of the University of Glasgow http://eprints.gla.ac.uk 


\title{
Green Hybrid Satellite Terrestrial Networks: Fundamental Trade-off Analysis
}

\author{
Jiaxin Zhang*, Barry Evans ${ }^{\dagger}$, Muhammad Ali Imran ${ }^{\dagger}$, Xing Zhang* and Wenbo Wang* \\ ${ }^{*}$ Wireless Signal Processing \& Networks Lab (WSPN) \\ Key Laboratory of Universal Wireless Communication \\ Beijing University of Posts and Telecommunications (BUPT), Beijing, China \\ Corresponding Author: Jiaxin Zhang, email: zhangjxbupt@gmail.com \\ $\dagger$ Institute for Communication Systems (ICS) \\ University of Surrey, Guildford, UK
}

\begin{abstract}
With the worldwide evolution of $4 \mathrm{G}$ generation and revolution in the information and communications technology (ICT) field to meet the exponential increase of mobile data traffic in the 2020 era, the hybrid satellite and terrestrial network based on the soft defined features is proposed from a perspective of 5G. In this paper, an end-to-end architecture of hybrid satellite and terrestrial network under the control and user Plane $(\mathrm{C} / \mathrm{U})$ split concept is studied and the performances are analysed based on stochastic geometry. The relationship between spectral efficiency (SE) and energy efficiency (EE) is investigated, taking consideration of overhead costs, transmission and circuit power, backhaul of gateway (GW), and density of small cells. Numerica results show that, by optimizing the key parameters, the hybrid satellite and terrestrial network can achieve nearly 90\% EE gain with only 3\% SE loss in relative dense networks, and achieve both higher EE and SE gain (20\% and 5\% respectively) in sparse networks toward the future $5 \mathrm{G}$ green communication networks.

Index Terms-Hybrid satellite and terrestrial network, $\mathrm{C} / \mathrm{U}$ split architecture, spectral and energy efficiency, 5G
\end{abstract}

\section{INTRODUCTION}

The next generation networks (5G) are expected to support an increasing number of connected devices and diversity of applications, which requires wireless communication systems to move towards a real information-and-user based network. In order to improve the manageability and adaptability of the network, the control and data plane separation based on the software orchestration mechanism has attracted considerable attention [1] [2], and is utilized here in the hybrid satellite terrestrial network.

Compared with the terrestrial network, satellite communications have quite a lot of advantages including coverage extension, content distribution, providing resilience and energy saving [3], so that the integration of satellite and terrestrial networks could become an important feature for 5G. the European Union has set up the 5G PPP (Public Private Partnership) research programme to fund researches from industry, academia and research organizations toward an integrated $5 \mathrm{G}$ standard [4]. Meanwhile, the future network evolves with the high exponentially growth of energy consumption, so that the indicator of EE has become of great importance [5], given the fact that the entire network infrastructure operates under a power constraint situation. In [6] the overall energy efficiency of the hybrid network is enhanced by a real-time adaptive transmission scheme.

The above mentioned works focus on the analysis of mobile satellite network performance. However, with the soft-defined features, the network architecture and key procedures have been quite different in $\mathrm{C} / \mathrm{U}$ split networks. To the best of our knowledge, the performance of the hybrid satellite terrestrial networks under the $\mathrm{C} / \mathrm{U}$ split architecture has not been studied where the satellite and the base stations cooperate in both $\mathrm{C}$ plane and U-planes.

The main differences of the hybrid $\mathrm{C} / \mathrm{U}$ split network with the traditional mobile satellite network can be summarized into following two respect. On the one hand, the C-plane satellite communication system with a global knowledge of terminals and base stations, including the context information and user behaviours, can be utilized to realize an informationcentric network (ICN) with a more flexible delivery strategy and dynamic bandwidth allocation schemes [7]. The terminals with dual-connection in hybrid networks can receive traffic from both satellite and small cells, alongwith better control and scheduling from the satellite. On the other hand, the signalling overheads can be reduced in the hybrid network in the U-plane, so that the small cells eNodeB (SeNBs) can benefit from larger capacity for data transmission and longer sleep periods [8]. Compared with the current LTE system, the public downlink control information, e.g., Physical Downlink Control Channel (PDCCH), Physical Hybrid-ARQ Indicator Channel (PHICH), Physical Control Format Indicator Channel (PCFICH), can be transmitted via the satellite to improve the available data transmission in the U-plane. In our previous work [9], it has been shown that the throughput and coverage can be enhanced by nearly $136 \%$ and $77 \%$ respectively in the $\mathrm{C} / \mathrm{U}$ split hybrid network compared with current LTE-A networks.

In this paper, based on our proposed end-to-end system model of hybrid satellite terrestrial networks with soft defined features, and the EE of the network is analysed based on stochastic geometry. The influence of overhead, transmission and circuit power, backhaul of gateway and density of SeNBs are evaluated to verify the trade-off between SE and EE in the $\mathrm{C} / \mathrm{U}$ split hybrid networks, which is a promising way towards future high-throughput green communication systems. 
The rest of this paper is organized as follows: Section II describes the proposed system model of end-to-end $\mathrm{C} / \mathrm{U}$ split satellite terrestrial network. The throughput, power consumption and energy efficiency are derived to study the influence of various key parameters in Section III. Simulation results are obtained and analysed in Section IV. Finally, conclusions are drawn in Section V.

\section{SYSTEM MODEL}

In this section, we propose an end-to-end hybrid satellite terrestrial network with control and user plane split, shown in Fig. 1. Under this architecture, the satellite is assumed as the home subscriber server (HSS) with the central database containing information about the network's subscribers and mobility management entity (MME) with signalling functions related to the mobility and security of the Evolved Universal Terrestrial Radio Access Network (E-UTRAN) access. Thus the satellite maintains seamless large-scale coverage in the Cplane and also for low rate data transmissions, e.g., machinetype-communication (MTC) service, in the U-plane for the terminals beyond the coverage of SeNBs, named primary users (PUEs). In the coverage of SeNBs with high frequency (e.g., $3.5 \mathrm{GHz}$ ), the secondary users (SUEs) also retain the C-plane link with the satellite whilst receiving high-quality mobile multimedia transmission from the SeNBs in the terrestrial network in the U-plane, so that the control channel and public signallings of the SeNBs can be significantly simplified.

However, the satellite is assumed to have limited computing ability, so that it is more realistic for the S/P-GW to process the related information and send back the calculated data via the satellite. Furthermore, the gateway also routes traffic in the terrestrial network, as well as C-plane control signallings and U-plane traffic for the satellite back to the external internet.

Based on mature stochastic geometry theory [10], SeNBs in the terrestrial network are modeled as independent homogeneous Poisson Point Process (PPP) $\Phi$ with a density of $\lambda_{b}$, which is the number of small cells in the coverage of the satellite narrow spot beam. The constant transmission power and bandwidth of satellite and SeNB are $P_{t s}, P_{t b}, W_{s}, W_{b}$ respectively. For a typical UE with a distance from the nearest SeNB $r$, the received power $P_{r b}$ can be modeled as $P_{t b} \mathrm{~h}_{t b} r^{-\alpha}$ where the standard power loss propagation model is used with path loss exponent $\alpha$ and iid Rayleigh fading on all links from SeNB are modeled as exponential distribution with mean $1 / u$ : $h_{t b} \sim \exp (u)$. Taking advantage of the PPP properties, the nearest distance for a typical UE to the small cell is:

$$
f_{r}(r)=e^{-\pi \lambda_{b} r^{2}} 2 \pi \lambda_{b} r
$$

The Low Earth Orbit (LEO) with low frequency is considered (e.g. $2 \mathrm{GHz}$ band) with the wavelength of $\lambda$. We also consider constellations of LEO satellites (altitude $d$ of around $1000 \mathrm{~km}$ ) as the delay is reduced and the UE power requirements are lower. In the future, it may be possible to use the constellations of high throughput satellite (HTS) in $\mathrm{Ka}$ or $\mathrm{Ku}$ band, but these are not considered herein. The LEO satellites employ large number of spot-beams (satellite cells)

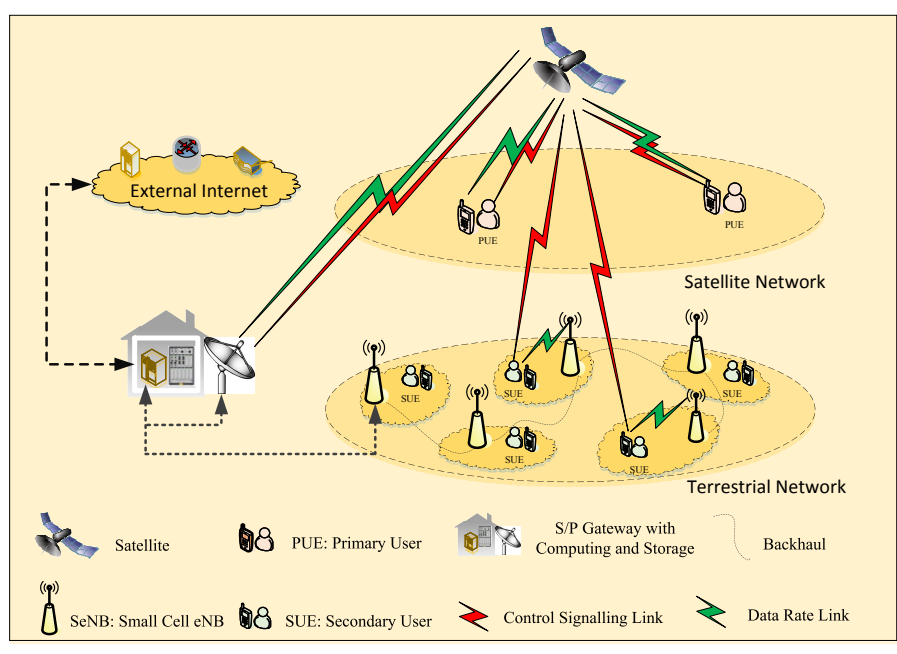

Fig. 1. End-to-end hybrid satellite terrestrial network with control and user plane separation.

within their coverage as well as frequency re-use between them. So co-channel interference is an issue but we consider that it is not a dominant parameter herein. The satellite spot beam handover will be designed into the constellation systems and need not be considered here. Doppler shift is also compensatable and is not considered. The latency issue drives us to consider LEO satellite and its affect on the C-plane siganallings is considered in other papers.

One of the spot beams coverage of the LEO is set as the study objective region in this model with the numerous SeNBs contained in it. In the satellite link, the received power is derived as:

$$
\mathrm{P}_{r s}=P_{t s} G_{t} G_{r} \frac{\lambda^{2}}{(4 \pi d)^{2} L},
$$

where $L$ is the atmospheric loss, $P_{t s}$ is the transmission power of satellite, $d$ is the altitude and $G_{t}, G_{r}$ are the typical antenna gains of transmitter and receiver in the downlink.

In the proposed $\mathrm{C} / \mathrm{U}$ split network, all of the UEs get in the C-plane connection via satellite, while the U-plane access strategy is based on the Reference Signal Receiving Power (RSRP). The SeNBs are configured with bias $\theta$, which can be used to adjust the probability of getting access to satellite or SeNBs. The access strategy in U-plane is given as follows :

$$
\left\{\begin{array}{c}
\theta \frac{P_{\mathrm{tb}} E\left[h_{t b}\right]}{r^{\alpha}}>\mathrm{P}_{r s}, \text { get access to the } \operatorname{SeN} B \\
\theta \frac{P_{\mathrm{tb}} E\left[h_{t b}\right]}{r^{\alpha}}<\mathrm{P}_{r s}, \text { get access to the satellite }
\end{array},\right.
$$

where $\frac{P_{\mathrm{tb}} E\left[h_{t b}\right]}{r^{\alpha}}$ is the RSRP in the terrestrial network. Then we suggest substitution of $\eta=\sqrt[\alpha]{\frac{\theta P_{\mathrm{tb}}}{u \mathrm{P}_{r s}}}$, deriving (4) as

$$
\left\{\begin{array}{c}
r<\eta, \text { get access to the SeNB } \\
r>\eta, \text { get access to the satellite }
\end{array} .\right.
$$

\section{ENERGY EFFICIENCY ANALYSIS}

In this section, we calculate analytically the throughput and energy consumption of the network and derive the SE and EE of the end-to-end hybrid satellite and terrestrial network. 


\section{A. Throughput}

Based on the model of stochastic geometry [10], for a typical UE, the small cell layer spectral efficiency $S E_{b}$ is:

$$
\begin{aligned}
& S E_{b}=E\left\{\log _{2}\left(1+S I N R_{b} \mid r\right) \times \mathrm{P}_{r o \_b}(r<\eta)\right\} \\
& =E\left\{\log _{2}\left(1+\frac{P_{t b} h_{t b} r^{-\alpha}}{\sigma^{2}+\sum_{b^{\prime} \notin \Phi / b 0} \frac{P_{t b^{\prime}} h_{t b^{\prime}}}{r^{\prime \alpha}}} \mid r\right)\right\} \times \int_{0}^{\eta} f_{r}(r) d r \\
& =\frac{1}{\ln 2} \cdot \int_{r>0} d r \int_{t>0} d t\left\{e^{\frac{-u r^{\alpha}}{P_{t b}}\left(e^{t}-1\right) \sigma^{2}}\right. \\
& \left.\cdot e^{-\pi \lambda_{\mathrm{b}} r^{2}\left(1+\left(e^{t}-1\right)^{2 / \alpha} \int_{\left(e^{t}-1\right)^{-2 / \alpha}}^{\infty} \frac{1}{1+x^{\alpha / 2}} d x\right)} 2 \pi \lambda_{\mathrm{b}} r\right\} .
\end{aligned}
$$

Neglecting the thermal noise $\sigma^{2}$ in the interference limited terrestrial network and assuming the path loss exponent $\alpha=4$ for all the links, the throughput of SeNBs in the U-plane in the terrestrial network can be simplified as follows :

$$
\begin{aligned}
& \text { Throughput }_{b}=\frac{1}{\ln 2} \cdot \lambda_{b} \times W_{b} \times\left(1-O_{\text {verhead }_{-}}\right) \\
& \times \int_{0}^{\infty} \int_{0}^{\pi \lambda_{b} \eta^{2}} e^{-v\left(1+\sqrt{\left(e^{t}-1\right)}\left(\frac{\pi}{2}-\arctan \left(\frac{1}{\sqrt{\left(e^{t}-1\right)}}\right)\right)\right)} d v d t,
\end{aligned}
$$

where the overhead of SeNBs $O_{\text {verhead_b }}$ in the U-plane is approximately $15 \%$ as evaluated in [8] due to the public broadcast information and signallings are diverted to the satellite.

The spectral efficiency of the satellite is given as:

$$
\begin{aligned}
& S E_{S}=\mathrm{E}\left\{\log _{2}\left[1+S I N R_{s} \mid r\right] \times \mathrm{P}_{r_{0} s}(r>\eta)\right\} \\
& =\mathrm{E}\left\{\log _{2}\left[1+\frac{\mathrm{P}_{r s}}{k T_{\text {on_earth }} W_{s}} \mid r\right]\right\} \times \int_{\eta}^{\infty} f_{r}(r) d r \\
& =\log _{2}\left(1+\frac{P_{t s} G_{t} G_{r} \lambda^{2}}{(4 \pi d)^{2} L k T_{\text {on_earth }} W_{s}}\right) \exp \left(-\pi \lambda_{b} \eta^{2}\right) .
\end{aligned}
$$

The thermal noise $k T_{\text {on_earth }} W_{s}$ should be taken into consideration because there is no interference between satellite and small cells, where $k$ is the Boltzmann constant $1.3806488 \times 10^{-23} \mathrm{~J} / \mathrm{K}$ and $T_{\text {on_earth }}$ is the noise temperature of terminal. Thus the throughput of satellite is:

$$
\begin{aligned}
& \text { Throughput }_{s}=W_{s} \times\left(1-O_{\text {verhead_s }}\right) \\
& \times \log _{2}\left(1+\frac{P_{t s} G_{t} G_{r} \lambda^{2}}{(4 \pi d)^{2} L k T_{\text {on_earth }} W_{s}}\right) \\
& \times \exp \left(-\pi \lambda_{b} \sqrt{\frac{P_{t b}}{P_{t s}} \cdot \frac{\theta}{\lambda^{2}} \cdot \frac{(4 \pi d)^{2} L}{G_{t} G_{r}}}\right),
\end{aligned}
$$

where the path loss exponent $\alpha=4$ is used and the overhead of satellite $O_{\text {verhead_s }}$ in the U-plane is about $15 \%$ given in [8]. The overall throughput in the U-plane in this hybrid network with $\mathrm{C} / \mathrm{U}$ split architecture is:

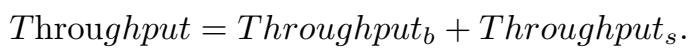

\section{B. Energy Consumption}

The overall energy consumption consists of two parts: the power consumption of the SCs and the satellite gateway ( $\mathrm{P}$ $\mathrm{GW}$ and S-GW). Although the satellite is operated by solar panels and batteries which are limited in energy capacity, the power of the satellite is not taken into consideration due to the fact that the solar energy is renewable, sustainable and not included in the grid power consumption, so that the oneoff energy to launch the satellite will be very small compared with the power consumed over the useful life. Furthermore, the power consumed in the terminal side can be ignored, because the access network (base stations) already accounts for nearly $80 \%$ of the overall power consumption. Also the power used for calculating and transmitting in cell phone are even smaller, compared with the power cost by the brightness of the screen and apps updating in the background.

The power model of SeNB is modeled as follows according to the reference [11]:

$$
P_{b}=\alpha^{\prime} P_{t b}+P_{b 0}
$$

where $P_{t b}$ is the transmission power related to the traffic load, $\alpha^{\prime}$ is the increase coefficient and $P_{b 0}$ is the static power of SeNB.

The gateway in the hybrid network plays an important role in the following three parts:

- Calculate and store the details about the subscribers and the related mobility management information

- Send back the processed information to the satellite in the uplink

- Route the traffic of the network to the external network

So the energy consumption of the gateway is calculated as the sum of the energy cost in three parts:

$$
P_{\text {gateway }}=P_{g t x}+P_{c}+P_{g b h},
$$

where $P_{g t x}$ is the uplink traffic transmission power from the gateway to the satellite. Taking advantage of the link budget equation, this part of the power consumption is given as:

$$
P_{g t x}=\frac{\left(2^{\text {Throughput }} / W_{\mathrm{g}}-1\right) \times \mathrm{kT}_{\text {on_satellite }} W_{\mathrm{g}}}{\frac{G_{t}^{\prime} G_{r}^{\prime} \lambda^{\prime 2}}{(4 \pi d)^{2} L^{\prime}}},
$$

where $W_{\mathrm{g}}$ is the bandwidth of gateway, $T_{\text {on_satellite }}$ and $\lambda^{\prime}$ are the noise temperature and uplink wavelength of LEO, and $G_{t}{ }^{\prime}, G_{r}{ }^{\prime}, L^{\prime}$ are the transmitter and receiver antenna gains, uplink atmosphere loss and rain attenuation respectively. The static computing power consumption $P_{c}$ is given in Table 3 in [12], and $P_{g b h}$ is the energy consumption of backhaul back to the external network [13]:

$$
P_{g b h}=\frac{\text { Throughput }_{b}+S E_{s} \times W_{s}}{100 M b p s} \times 50 W .
$$

\section{Spectral and Energy Efficiency}

The SE refers to the U-plane data rate that can be transmitted over a given bandwidth in the whole system:

$$
S E=\frac{\text { Throughput }}{\lambda_{b} W_{b}+W_{s}} .
$$

The hybrid network energy efficiency is defined as the ratio of downlink average throughput in the U-plane of the $\mathrm{C} / \mathrm{U}$ split network to total power consumption in the end-to-end system:

$$
E E=\frac{\text { Throughput }}{\lambda_{b} P_{b}+P_{\text {gateway }}} .
$$




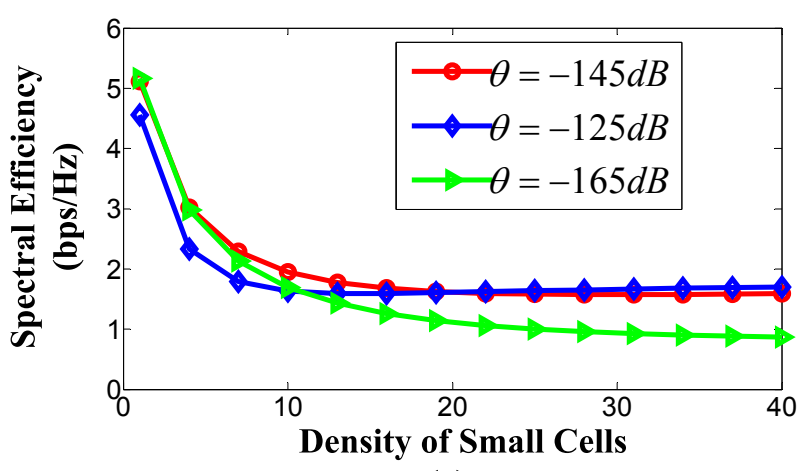

(a)

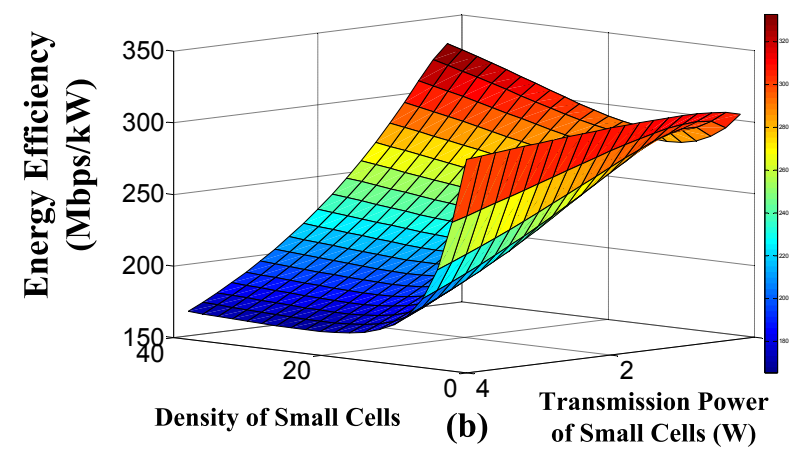

Fig. 2. SE and EE results: (a) SE vs. $\lambda_{b}$ under various bias $\theta$; (b) EE vs. $\lambda_{b}$ and $P_{t b}$ of SeNB;

\section{NUMERICAL RESULTS}

In this section, numerical results of the throughput, $\mathrm{SE}$ and $\mathrm{EE}$ of the hybrid satellite terrestrial network with $\mathrm{C} / \mathrm{U}$ split are presented. Typical values [14], such as $P_{t s} G_{t}=54.4 \mathrm{dBW}$, $W_{s}=30 \mathrm{MHz}, \lambda=137.3 \mathrm{~mm}, G_{r}=0 \mathrm{~dB}, d=1000 \mathrm{~km}$, $L=L^{\prime}=0 d B, T_{\text {on_earth }}=290 \mathrm{~K}$, are used to model the LEO channel link. For the terrestrial network, the key parameters are $W_{b}=10 \mathrm{MHz}, \alpha^{\prime}=16, P_{b 0}=28.7 \mathrm{~W}$, $P_{t b}=0 \sim 4 W, u=1$, according to the Earth Project. For the gateway uplink transmission, the computing consumption $P_{c}=355 \mathrm{~W}, W_{g}=15 \mathrm{MHz}, T_{\text {on_satellite }}=26 \mathrm{dBK}$, $\lambda^{\prime}=50 \mathrm{~mm}, G_{t}^{\prime}=40 \mathrm{~dB}, G_{r}^{\prime}=16 \mathrm{~dB}$, where C-band $6 \mathrm{GHz}$ uplink channel with a $2 \mathrm{~m}$ antenna at the gateway.

The theoretical analysis of the network performance is illustrated in Fig 2. The bias $\theta$ significantly influences the probability for the UE to get access to the satellite or small cell, and the density of small cells $\lambda_{b}$ have quite different impact on the SE of the network, shown Fig 2. (a). With small bias, e.g. $\theta=-165 d B$, the UE can hardly get access to SeNBs, so that the performance degrades quickly with the increase of $\lambda_{b}$. As a consequence, the advantages of the satellite will weaken with large bias, e.g. $\theta=-125 d B$. In this way, a proper bias with median value of $\theta=-145 \mathrm{~dB}$, should be used to gain benefit from both the small cell and the satellite. The constant path loss factor is reflected by the bias, so that the absolute value is small. Note that in terrestrial macro cell comes the bias is around $0 \mathrm{~dB}$, but here due to the lower received power from the satellite we have a much smaller value. Though the received power is low in the satellite network, the SINR could also be larger than that in the terrestrial network, because of the severe interference between small cells.

The 3-D figure is utilized to illustrate the relationship between EE and the key parameters in Fig. 2 (b). On the one hand, the EE increases with the density of small cells, initially benefiting from larger throughput and relatively lower constant power consumption, and then remains constant because the throughput and the energy consumption grow simultaneously. On the other hand, the EE shows an initial upward trend and then reduces drastically with the transmission power of the SeNB $P_{t b}$, because of the larger SE and power consumption respectively.

Under the $\mathrm{C} / \mathrm{U}$ split hybrid network, there exists a fundamental trade-off with the SE and EE, shown in Fig. 3. In sparse networks, shown in Fig. 3 (a), when $\lambda_{b}$ is small and most of the terminals get access to the satellite, resulting in SE reduction with $P_{t b}$. At the same time, EE rises first and then reduces with $P_{t b}$ due to the effect of the gateway and small cell power consumption. Compared with the network with maximum $P_{t b}=4 \mathrm{~W}$, the median value of $P_{t}$ (e.g., $0.5 \mathrm{~W}$ ) could achieve nearly $20 \%$ EE gain and $5 \%$ SE gain simultaneously in this relatively sparse network (e.g., $\lambda_{b}=3$ ).

In relative dense network, shown in Fig. $3(\mathrm{~b})$, larger $P_{t b}$ helps to increase SE and EE initially, but then EE drops quickly and SE remains stable with the increase of $P_{t b}$. In thses relatively dense networks (e.g., $\lambda_{b}=40$ ), compared with the SE optimized parameter setting, proper transmission power $P_{t}$ (e.g., $0.5 W$ ) helps to increase EE by approximately $90 \%$ with only $3 \%$ SE loss.

In sparse networks, the satellite is able to maintain coverage for users, especially in remote places. In addition the advantages of the satellite bandwidth can be used to improve the throughput hugely while maintaining the energy consumption. Thus the trade-off between SE and EE in sparse networks can be utilized to increase EE significantly with very slight SE decrease.

The delay of the hybrid system is of great importance in future $5 \mathrm{G}$ networks. In the hybrid $\mathrm{C} / \mathrm{U}$ split network, the oneway U-plane latency is about $2.6 \mathrm{msec}$ and the satellite is assumed to forward the processing information to the gateway before transmitting each response to the UE requests. In addition to that, a processing delay of $4 \mathrm{msec}$ is considered at the $\mathrm{UE}$, the gateway and the SeNBs. Finally, the processing delay at the satellite is considered to be $60 \mathrm{msec}$ before transmitting any information either to the UE or to the gateway. Thus the delay from power on to RRC Connection is nearly $280 \mathrm{msec}$, which is higher than the LTE network.

However, it is worth mentioning that the part of the C-Plane that is being separated from the U-Plane represents only the control signalling for the UE to maintain connection with the network (Broadcasting Information). Thus, the rest part of the C-Plane, that is responsible to provide the support and the reliability of the transmitted data, including Downlink Control Information (DCI) and Reference Signals (RS), is still maintained in the U-Plane ( nearly $15 \%$ for the hybrid architecture). 


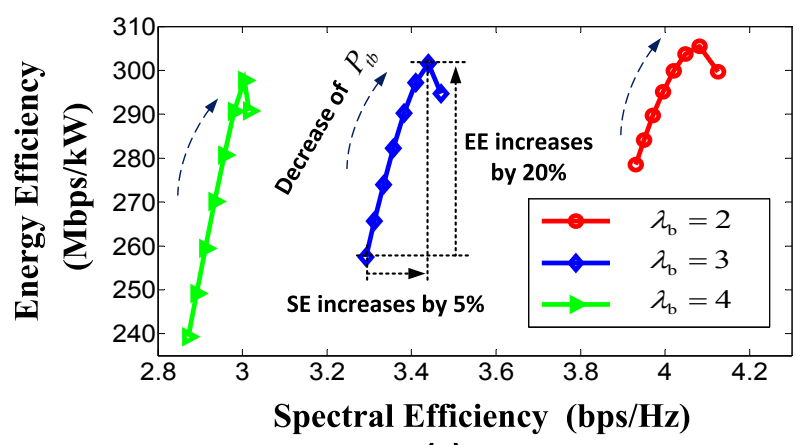

(a)

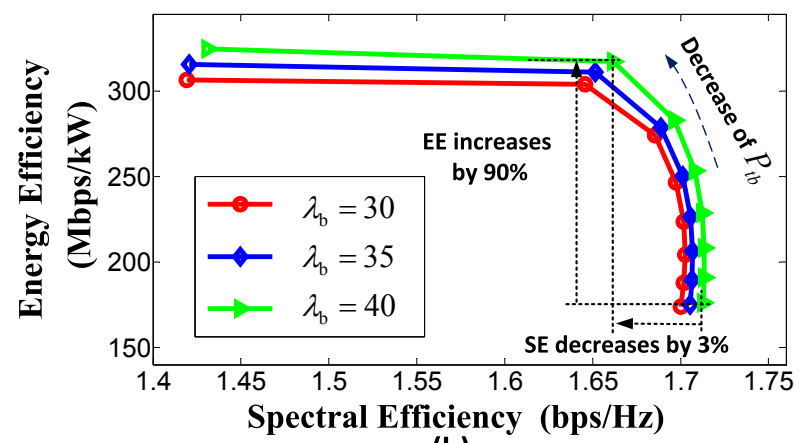

(b)

Fig. 3. Tradeoff between SE and EE: (a) SE vs. EE in under various $\lambda_{b}$ in sparse network; (b) SE vs. EE in under various $\lambda_{b}$ in dense network.

Thus the traffic scheduling and resource allocation in U-plane is not affected and the proposed architecture not influences the sense of "delay" that the user feels.

From the aspect of standard, timer values of LTE (Timers $\mathrm{T} 300, \mathrm{~T} 301)$ for the reception of particular messages in Cplane (4 times of request delay ranges $400-8000 \mathrm{msec}$ ) can be met by this hybrid network. However, the latency from RRC_IDLE to RRC_CONNECTED timer is with maximum value of $100 \mathrm{msec}$ can not be met. A suggestion is to develop on-board processing satellite payloads, which is a very promising study area, to reduce the satellite processing delay to approximately $40 \mathrm{msec}$.

\section{CONClusion And Future Work}

In this paper, the performances of end-to-end $\mathrm{C} / \mathrm{U}$ split hybrid satellite and terrestrial network, in terms of energy efficiency, are investigated. Taking consideration of key parameters, e.g. density of small cells, overhead cost, transmission power, circuit power, as well as the influence of the gateway, theoretical analyse of SE and EE are discussed. Numerical results are verified the fundamental trade-off between SE and $\mathrm{EE}$, so that the $5 \mathrm{G} \mathrm{C/U}$ split hybrid network can be optimized with both higher SE and EE by adjusting the related key parameters. In relatively dense network, 3\% of SE loss can bring nearly $90 \%$ energy efficiency improvement toward the future $5 \mathrm{G}$ green communication networks. The latency of the proposed architecture is discussed and suggestions are given in the end. In future, various U-plane scheduling strategies focusing on various QoS requirements will be studied based on the soft defined features of the hybrid network, and multiple key indicators, such as QoE, delay, security, will be further analysed. The constellation of HTS satellite in $\mathrm{Ka}$ or $\mathrm{Ku}$ band will be studied as part of the integrated $5 \mathrm{G}$ networks with millimetre waves used in dense small cells as a logical extension of the work provided herein.

\section{ACKNOWLEDGEMENT}

This work is supported by National 973 Program under grant 2012CB316005, the National Science Foundation of China (NSFC) under grant 61372114, the Fundamental Research Funds for the Central Universities under grant 2014ZD03-01, the New Star in Science and Technology of Beijing Municipal Science \& Technology Commission (Beijing Nova Program: Z151100000315077), the Beijing Higher Education Young Elite Teacher Project under grant YETP0434, and the China Scholarship Council.

\section{REFERENCES}

[1] Ericsson White paper: 5G energy performance - key technologies and design principles, http://www.ericsson.com/res/docs/whitepapers/wp-5genergy-performance.pdf, April 2015.

[2] X. Zhang, J. Zhang, W. Wang, Y. Zhang, C.-L. I, Z. Pan, G. Li, Y. Chen, "Macro-assisted data-only carrier for 5G green cellular systems," IEEE Communications Magazine, vol. 53, no. 5, pp. 223-231, May 2015.

[3] B. G. Evans, "The role of satellites in 5G," 2014 7th Advanced Satellite Multimedia Systems Conference and the 13th Signal Processing for Space Communications Workshop, pp. 197-202, Sep. 2014.

[4] EC H2020 5G Infrastructure PPP Pre-structuring Model RTD \& INNO Strands, http://5g-ppp.eu.

[5] F. Alagoz, G. Gur, "Energy Efficiency and Satellite Networking: A Holistic Overview," Proceedings of the IEEE, vol. 99, no. 11, pp. 19541979, Nov. 2011

[6] S. Kandeepan, K. Gomez, L. Reynaud, T. Rasheed, "Aerial-terrestrial communications: terrestrial cooperation and energy-efficient transmissions to aerial base stations," IEEE Transactions on Aerospace and Electronic Systems, vol. 50, no. 4, pp. 2715-2735, Oct. 2014.

[7] Y. Kawamoto, Z. Fadlullah, H. Nishiyama, N. Kato, M. Toyoshima, "Prospects and challenges of context-aware multimedia content delivery in cooperative satellite and terrestrial networks," IEEE Communications Magazine, vol. 52, no. 6, pp. 55-61, June 2014.

[8] T. Spathopoulos, O. Onireti, A.H. Khan, M. Imran, K. Arshad, "Hybrid Cognitive Satellite Terrestrial Coverage: A case study for 5G deployment strategies", 10th International Conference on Cognitive Radio Oriented Wireless Networks (CROWNCOM 2015), 21-23 April 2015.

[9] J. Zhang, B. Evans, M. A. Imran, X. Zhang and W. Wang, "Performance Analysis of C/U Split Hybrid Satellite Terrestrial Network for 5G Systems", IEEE 20th International Workshop on Computer Aided Modeling and Design of Communication Links and Networks (CAMAD), 2015.

[10] J. G. Andrews, F. Baccelli, R. K. Ganti, "A Tractable Approach to Coverage and Rate in Cellular Networks," IEEE Transactions on Communications, vol. 59, no. 11, pp. 3122-3134, Nov. 2011.

[11] G. Auer, V. Giannini, C. Desset, I. Godor, P. Skillermark, M. Olsson, M. A. Imran, D. Sabella, M. J. Gonzalez, O. Blume, A. Fehske, "How much energy is needed to run a wireless network?," IEEE Wireless Communications, vol. 18, no. 5, pp. 40-49, October 2011.

[12] J. Baliga, R. W. A. Ayre, K. Hinton, R. S. Tucker, "Green Cloud Computing: Balancing Energy in Processing, Storage, and Transport," Proceedings of the IEEE, vol. 99, no. 1, pp. 149-167, Jan. 2011.

[13] A. J. Fehske, P. Marsch, G. P. Fettweis, "Bit per Joule efficiency of cooperating base stations in cellular networks," 2010 IEEE GLOBECOM Workshops (GC Wkshps), pp. 1406-1411, Dec. 2010

[14] C. Qian, S. Zhang, W. Zhou, "Traffic-based dynamic beam coverage adjustment in satellite mobile communication," 2014 Sixth International Conference on Wireless Communications and Signal Processing (WCSP), pp. 1-6, Oct. 2014. 